\title{
Elastic Moduli of Ultrathin Amorphous Polymer Films
}

\author{
Christopher M. Stafford, ${ }^{*}, \dagger$ Bryan D. Vogt, ${ }^{\dagger}$ Christopher Harrison, ${ }^{\dagger, \S}$ \\ Duangrut Julthongpiput, ${ }^{\dagger, \perp}$ and Rui Huang
}

Polymers Division, National Institute of Standards and Technology, Gaithersburg, Maryland 20899, and Department of Aerospace Engineering and Engineering Mechanics, University of Texas, Austin, Texas 78712

Received April 7, 2006; Revised Manuscript Received June 2, 2006

\begin{abstract}
The elastic moduli of ultrathin poly(styrene) (PS) and poly(methylmethacrylate) (PMMA) films of thickness ranging from $200 \mathrm{~nm}$ to $5 \mathrm{~nm}$ were investigated using a buckling-based metrology. Below $40 \mathrm{~nm}$, the apparent modulus of the PS and PMMA films decreases dramatically, with an order of magnitude decrease compared to bulk values for the thinnest films measured. We can account for the observed decrease in apparent modulus by applying a composite model based on the film having a surface layer with a reduced modulus and of finite thickness. The observed decrease in the apparent modulus highlights issues in mechanical stability and robustness of sub-40 $\mathrm{nm}$ polymer films and features.
\end{abstract}

Introduction. There are numerous technological drivers for the use of thin (sub-1 $\mu \mathrm{m}$ ) and ultrathin (sub-100 nm) polymer films and features. The stability and reliability of these confined polymer systems are critical to numerous existing and emerging technologies such as next-generation lithography, lubricating coatings, sensors, and organic electronics. It is widely accepted that the physical properties of thin polymer films can deviate substantially from their bulk counterparts. ${ }^{1,2}$ However, most experimental studies have focused on elucidating the thickness dependence of the thermal properties in thin polymer films, specifically the glass transition temperature $\left(T_{\mathrm{g}}\right)$, as a function of interfacial interactions and molecular entanglements, often with conflicting results. ${ }^{1}$ From a nanomanufacturing perspective, a direct measure of the mechanical robustness (e.g., response to deformation) of confined polymers films would be extremely helpful to determine whether the mechanical properties display similar deviations from bulk response. For example, it has been shown that the lack of mechanical strength in polymer nanostructures leads to deformation and subsequent collapse of the structures when subjected to capillary forces induced during solvent evaporation. ${ }^{3}$ While a number of experimental methods are available for measuring the mechanical properties of thin polymer films, including methods based on indentation, ${ }^{4,5}$ surface acoustic waves, ${ }^{6}$ and beam curvature, ${ }^{7}$ adapting these measurement techniques to ultrathin polymer films remains challenging. To completely understand how confinement affects the mechanical properties and response of polymeric materials, it is critical that a range of experimental and theoretical methods for measuring the mechanical properties of ultrathin films as a function of film thickness be made available.

Recent molecular dynamics simulations of deformation in polymer nanostructures suggest that the elastic modulus of these structures remains bulklike for thicknesses down to $\approx 40 \mathrm{~nm}$. Below this critical threshold, the simulations predict that the apparent elastic modulus decays dramatically from bulk value

\footnotetext{
* Corresponding author: Fax +1 (301) 975-4924; e-mail chris.stafford@ nist.gov.

National Institute of Standards and Technology.

University of Texas.

$\S$ Present address: Sensor Physics Department, Schlumberger-Doll Research, Ridgefield, CT 06877.

${ }^{\perp}$ Present address: Assembly Technology Development, Intel Corporation, Chandler, AZ 85226.
}

as the thickness is decreased further, reaching $10 \%$ of the bulk value for a thickness of $\approx 7.5 \mathrm{~nm}$. Similar results were obtained using strain-fluctuation simulations, ${ }^{9}$ which circumvent the necessity that continuum mechanics still be valid at such small length scales. It was also concluded that the elastic constants become anisotropic in polymer nanostructures. Furthermore, recent nonequilibrium simulations ${ }^{10}$ demonstrate that local dynamic mechanical properties of polymer thin films decrease due to the presence of mechanically soft layers near the free surface of the film. These results are consistent with previous molecular dynamics simulations of the free surface in glassy polymer films, where a $1.5 \mathrm{~nm}$ thick region of enhanced mobility was observed. ${ }^{11}$

There are also recent examples in the literature of experimental methods for measuring the mechanical properties of thin and ultrathin polymer films, ${ }^{5,12,13}$ the most prevalent being Brillouin light scattering (BLS). BLS has been used to measure the elastic constants of both supported ${ }^{14,15}$ and free-standing ${ }^{16}$ polymer films and features with thicknesses from $375 \mathrm{~nm}$ down to $29 \mathrm{~nm}$, and it was observed that the high-frequency mechanical properties did not significantly change at these dimensions. Probing thinner free-standing films with BLS becomes difficult due to sample preparation and handing issues, while BLS measurements on thinner supported films are complicated by the presence of the substrate interface. To overcome this issue, a multilayer geometry was employed to generate alternating interfaces between mechanically dissimilar materials, poly(styrene) and poly(isoprene). ${ }^{17}$ BLS results from this geometry indicate no deviation in the mechanical properties of either material down to layer thicknesses of at least $16 \mathrm{~nm}$. However, there are no free interfaces in such a multilayer geometry; thus, any enhanced mobility at the air interface is negated. In contrast, wafer curvature experiments have been used to measure the low-frequency mechanical properties of supported ultrathin polymer films. ${ }^{7}$ From these experiments, it was suggested that the biaxial modulus of a $10 \mathrm{~nm}$ poly(styrene) film decreases by an order of magnitude from the bulk value. Direct measurements of the mechanical properties of supported thin polymer films using contact techniques such as indentation and atomic force microscopy are convoluted by the presence of the stiff substrate and thus are prohibitively difficult to perform at this time. 

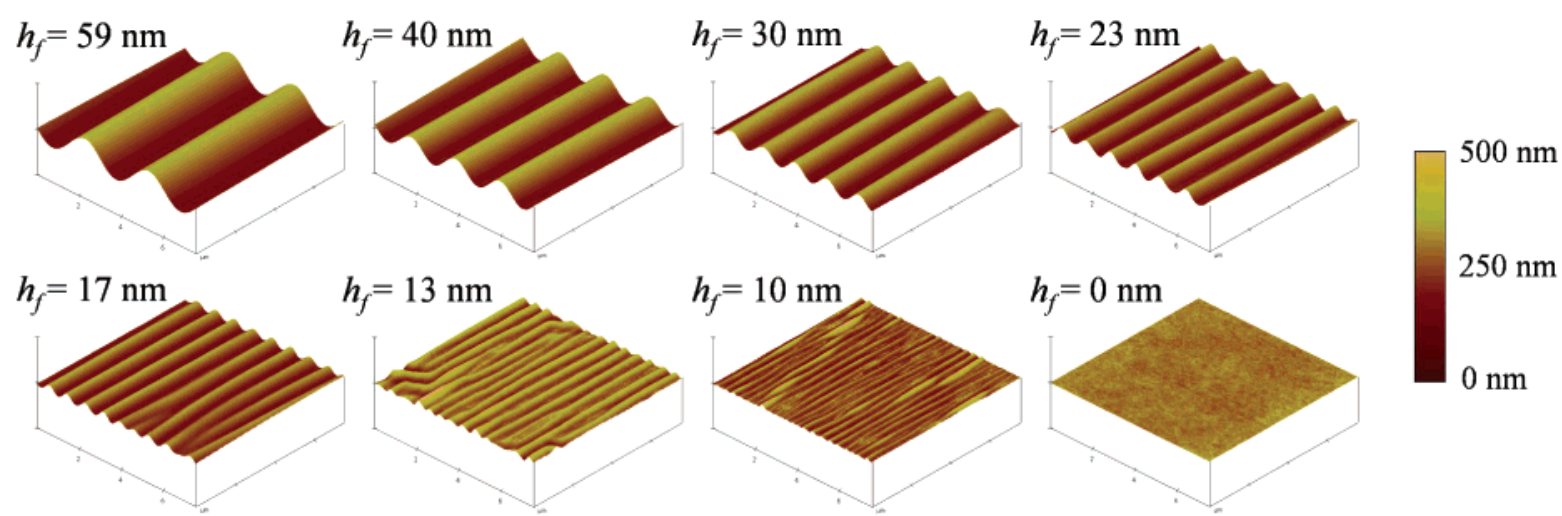

Figure 1. AFM height images of buckling patterns of PS films $\left(M_{\mathrm{w}}=1800 \times 10^{3} \mathrm{~g} / \mathrm{mol}\right)$ supported on PDMS for a series of film thicknesses $\left(h_{\mathrm{f}}\right)$. For all images, the scan size is $7.5 \mu \mathrm{m} \times 7.5 \mu \mathrm{m}$ and the height scale is $500 \mathrm{~nm}$.

In this Article, we illustrate a distinctive approach for measuring the mechanical properties of thin and ultrathin polymer films. We apply our recently reported buckling-based metrology ${ }^{18}$ to measure the elastic properties of polymer films with thicknesses ranging from 200 to $5 \mathrm{~nm}$. Here, a polymer film is adhered to a relatively soft elastic substrate and subjected to a small uniaxial compressive strain. From an energetic point of view, there are three competing terms associated with this geometry: membrane strain energy in the film, bending energy of the film, and near-surface strain energy in the substrate. Long wavelengths are suppressed due to the large strain energy associated with deformation of the soft substrate; short wavelengths are suppressed due to the sizable bending energy associated with the stiff film. Thus, the system undergoes periodic buckling with an intermediate wavelength to minimize the total strain energy. For an elastic film on an elastic substrate, the equilibrium wavelength, $\lambda_{\mathrm{e}}$, of buckling is given by ${ }^{19-21}$

$$
\lambda_{\mathrm{e}}=2 \pi h_{\mathrm{f}}\left(\frac{\bar{E}_{\mathrm{f}}}{3 \bar{E}_{\mathrm{s}}}\right)^{1 / 3}
$$

where $h_{\mathrm{f}}$ is the film thickness, $\bar{E}=E /\left(1-v^{2}\right)$ is the planestrain modulus, $E$ is Young's modulus, and $v$ is Poisson's ratio. The subscripts $\mathrm{f}$ and $\mathrm{s}$ denote film and substrate, respectively. Equation 1 can be rearranged to solve for the modulus of the film:

$$
\bar{E}_{\mathrm{f}}=3 \bar{E}_{\mathrm{s}}\left(\frac{\lambda_{\mathrm{e}}}{2 \pi h_{\mathrm{f}}}\right)^{3}
$$

In this derivation, the buckling wavelength is independent of the compressive strain as long as a critical strain is surpassed and the system remains linear-elastic. ${ }^{21,22}$ It should also be noted that the stress and strain in the substrate decays exponentially from the interface, with a decay length proportional to the wavelength. Consequently, the strain energy in the substrate becomes independent of the substrate thickness when the thickness is much greater than the wavelength; therefore, the substrate can be treated as a semiinfinite half space. Thus, by coupling a film of known thickness to a soft elastic substrate of known modulus, the compression-induced buckling wavelength can be related directly to the elastic modulus of the film.

Experimental Section. Polystyrene (PS) was purchased from Aldrich and used as received. Two different molecular weights of PS were investigated $\left(M_{\mathrm{w}}=114 \times 10^{3} \mathrm{~g} / \mathrm{mol}, M_{\mathrm{w}} / M_{\mathrm{n}}=1.1\right.$ and $\left.M_{\mathrm{w}}=1800 \times 10^{3} \mathrm{~g} / \mathrm{mol}, M_{\mathrm{w}} / M_{\mathrm{n}}=1.1\right)$, both of which are well above the entanglement molecular weight $\left(M_{\mathrm{e}}\right)$ of PS $\left(M_{\mathrm{e}}\right.$ $\left.\approx 13 \times 10^{3} \mathrm{~g} / \mathrm{mol}\right) .{ }^{23}$ In addition, poly(methyl methacrylate) $\left(\right.$ PMMA) $\left(M_{\mathrm{w}}=184 \times 10^{3} \mathrm{~g} / \mathrm{mol}, M_{\mathrm{w}} / M_{\mathrm{n}}=1.15, M_{\mathrm{e}} \approx 10 \times\right.$ $\left.10^{3} \mathrm{~g} / \mathrm{mol}\right)^{23}$ films were also studied. The PMMA was purchased from Scientific Polymer Products and used as received. All solutions were filtered through a $0.45 \mu \mathrm{m}$ Acrodisc filter prior to spin-coating. Poly(dimethylsiloxane) (PDMS) (Sylgard 184, Dow Chemical Co., Midland, MI) ${ }^{24}$ was hand-mixed at a ratio of 10:1 base monomer to curing agent and cast onto glass plates. The mixture was left at room temperature to allow trapped air bubbles to escape and then cured at $70{ }^{\circ} \mathrm{C}$ for $2 \mathrm{~h}$. After cooling, the PDMS was cut into $75 \mathrm{~mm} \times 25 \mathrm{~mm}$ specimens.

To prepare samples for testing, polymer films were transferred from silicon wafers to the surface of PDMS substrates as described previously, ${ }^{18}$ and a compressive strain was mechanically applied to the specimen. Specifically, thin films of PS and PMMA were first spin-coated from dilute toluene solutions onto polished silicon wafers. Film thickness was controlled via solution concentration $(0.1-3.0 \%$ by mass polymer) and spin speed $(1250-4000 \mathrm{rpm})$. Film thickness was measured by X-ray reflectivity (XR). Details of the reflectometer ${ }^{25}$ and reflectometry $^{26}$ are provided elsewhere. The roughness of the films, quantified by XR, ranged between 0.5 and $1.5 \mathrm{~nm}$ and exhibited no systematic dependence on film thickness. ${ }^{27}$ Films were transferred onto prestrained PDMS substrates via a water immersion technique. ${ }^{18}$ The PDMS substrates were prestrained by mounting onto a custom-built strain stage fitted with a mechanical actuator. ${ }^{28}$ After the film had been transferred to the PDMS surface, the strain was released almost instantaneously $(0.5 \mathrm{~s})$ by releasing the locking mechanism on the strain stage and allowing the PDMS to return to its original length. The magnitude of compressive strain was constant $(\epsilon=2.5 \%)$ for all films and thicknesses examined. In all experiments, buckling was induced at ambient temperature $(T=294 \pm 3$ $\mathrm{K})$, and all measurements of the buckling wavelength were also conducted at room temperature. The modulus of the PDMS substrates was measured on a Texture Analyzer (model TA.XT2i, Texture Technologies Corp., Scarsdale, NY) in tension mode.

Atomic force microscope (AFM) images were acquired on a Digital Instruments Dimension 3100 with a Nanoscope IV controller (Veeco Instruments, Woodbury, NY) operating in tapping mode. An average of 10 images per sample were collected over a $4 \mathrm{~h}$ period of time. Image analysis was conducted using the AFM software as well as a custom script written in IDL.

Results and Discussion. Figure 1 displays a series of tapping mode atomic force microscopy (AFM) micrographs that illustrate the decreasing wavelength as a function of decreasing film thickness as predicted by eq 1 . Note the buckling pattern can be reasonably described as a sinusoidal wave, which is an 


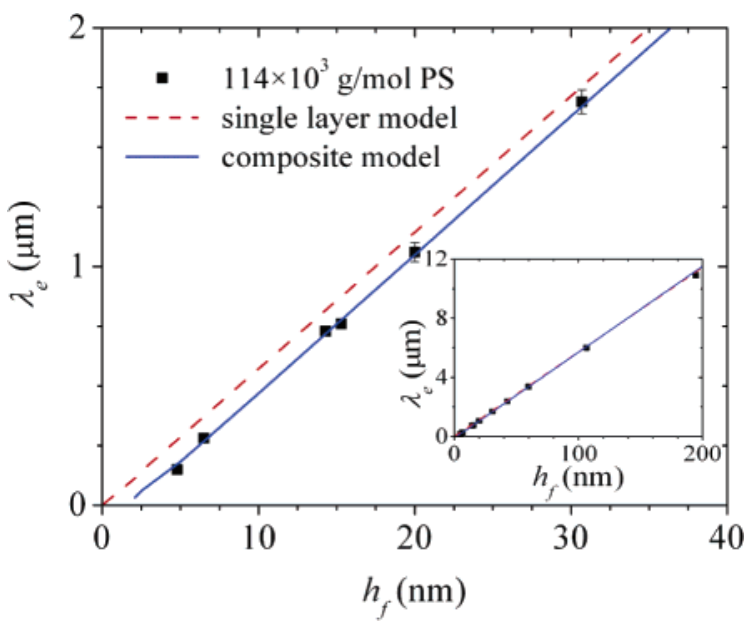

Figure 2. Measured wavelength $\left(\lambda_{\mathrm{e}}\right)(\mathbf{\square})$ as a function of film thickness $\left(h_{\mathrm{f}}\right)$ for PS films having $M_{\mathrm{w}}=114 \times 10^{3} \mathrm{~g} / \mathrm{mol}$. The dashed red line is eq 1 for a single-layer film $\left(\bar{E}_{\mathrm{f}}=4.0 \mathrm{GPa}\right)$; the discrepancy between this dashed line and the data demonstrates the breakdown in the analytical solution for the thinnest films. The solid blue line is the calculated wavelength that would originate from a composite model (eq 5) of a thin film consisting of a bulk $\left(\bar{E}_{\mathrm{f}}=4.2 \mathrm{GPa}\right)$ and surface layer $\left(\bar{E}_{\mathrm{f}}^{*}=0.1 \mathrm{GPa}, \delta=2 \mathrm{~nm}\right)$, demonstrating much better agreement with data. The inset displays the entire thickness range studied, but the deviations are more pronounced in the thinner films (main graph).

Table 1. Buckling Wavelength $\left(\lambda_{\mathrm{e}}\right)$ as a Function of Film Thickness $\left(h_{\mathrm{f}}\right)^{45}$ for PS and PMMA Materials Studied

\begin{tabular}{|c|c|c|c|c|c|}
\hline \multicolumn{2}{|c|}{$\begin{array}{c}114 \times 10^{3} \mathrm{~g} / \mathrm{mol} \\
\text { PS }\end{array}$} & \multicolumn{2}{|c|}{$\begin{array}{c}1800 \times 10^{3} \mathrm{~g} / \mathrm{mol} \\
\text { PS }\end{array}$} & \multicolumn{2}{|c|}{$\begin{array}{c}184 \times 10^{3} \mathrm{~g} / \mathrm{mol} \\
\text { PMMA }\end{array}$} \\
\hline$h_{\mathrm{f}}(\mathrm{nm})$ & $\lambda_{\mathrm{e}}(\mu \mathrm{m})$ & $h_{\mathrm{f}}(\mathrm{nm})$ & $\lambda_{\mathrm{e}}(\mu \mathrm{m})$ & $h_{\mathrm{f}}(\mathrm{nm})$ & $\lambda_{\mathrm{e}}(\mu \mathrm{m})$ \\
\hline 194.7 & $10.90 \pm 0.10$ & 174.7 & $9.14 \pm 0.13$ & 116.1 & $5.17 \pm 0.14$ \\
\hline 106.6 & $6.00 \pm 0.07$ & 96.6 & $5.11 \pm 0.11$ & 81.9 & $3.67 \pm 0.11$ \\
\hline 59.7 & $3.37 \pm 0.12$ & 59.2 & $3.13 \pm 0.09$ & 46.0 & $2.00 \pm 0.07$ \\
\hline 42.6 & $2.37 \pm 0.12$ & 40.4 & $2.15 \pm 0.07$ & 34.6 & $1.41 \pm 0.03$ \\
\hline 30.7 & $1.69 \pm 0.05$ & 29.7 & $1.56 \pm 0.05$ & 23.7 & $0.99 \pm 0.02$ \\
\hline 20.0 & $1.06 \pm 0.04$ & 22.6 & $1.14 \pm 0.05$ & 10.8 & $0.38 \pm 0.02$ \\
\hline 15.3 & $0.76 \pm 0.02$ & 17.6 & $0.86 \pm 0.03$ & 6.7 & $0.21 \pm 0.02$ \\
\hline 14.3 & $0.73 \pm 0.02$ & 12.9 & $0.59 \pm 0.02$ & & \\
\hline 6.5 & $0.28 \pm 0.01$ & 9.7 & $0.42 \pm 0.01$ & & \\
\hline 4.8 & $0.15 \pm 0.01$ & 5.0 & $0.14 \pm 0.01$ & & \\
\hline
\end{tabular}

assumption in the derivation of eq 1 . The amplitude of the buckling pattern decreases as a function of film thickness as expected from buckling mechanics for constant applied strain. In Table 1, we report the buckling wavelength as a function of film thickness for all materials studied. A cursory examination of the PS data reveals that the wavelength deviates from the behavior predicted by eq 1 for a constant modulus, as illustrated in Figure 2. Equation 1 (dashed red line) overestimates the data for film thicknesses less than $40 \mathrm{~nm}$. The inset in Figure 2, spanning a much greater range of thickness, shows that we recover the expected linear behavior in the thicker films. This behavior is found for both molecular weights studied. The dashed line in Figure 2 was generated for a film having $\bar{E}_{\mathrm{f}}=$ 4.0 GPa, a reasonable estimate that fits the thick film data well. Furthermore, the measured wavelength does not traverse the origin, as one would expect, but rather exhibits an intercept at film thickness $h_{\mathrm{f}} \approx 2 \mathrm{~nm}$. This suggests that films thinner than $2 \mathrm{~nm}$ would be stable and would not undergo buckling $\left(\lambda_{\mathrm{e}}=\right.$ 0 ). Interestingly, the Kuhn length of PS is $2 \mathrm{~nm},{ }^{29,30}$ but it is unclear how this characteristic length scale might factor into the mechanical behavior of ultrathin amorphous polymer films.

Similarly, we can use eq 2 to convert the PS data in Table 1 to an apparent plane-strain modulus $\left(\bar{E}_{\mathrm{f} \text {,app }}\right)$, as shown in Figure 3. The first salient feature of these data is the presence of a

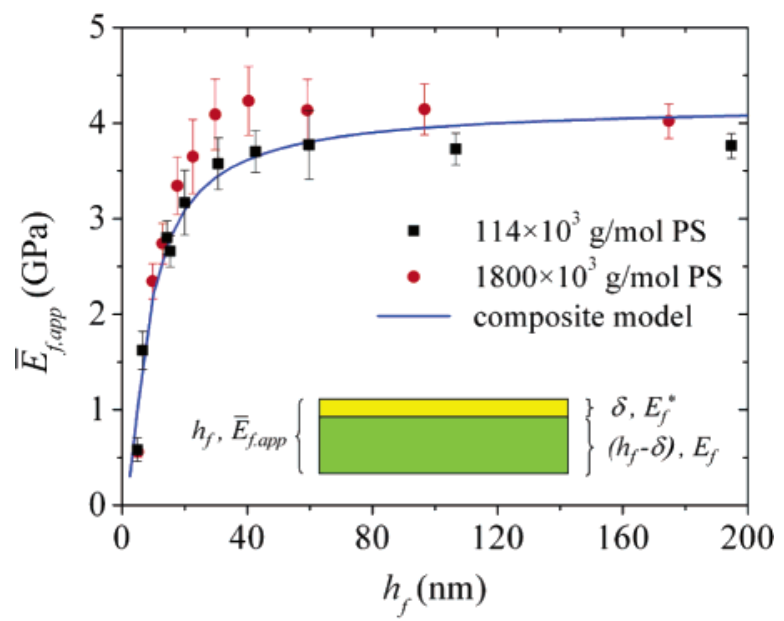

Figure 3. Apparent modulus $\left(\bar{E}_{\mathrm{f}, \mathrm{app}}\right)$ as a function of thickness $\left(h_{\mathrm{f}}\right)$ for PS films having $M_{\mathrm{w}}=114 \times 10^{3} \mathrm{~g} / \mathrm{mol}(\mathbf{\square})$ and $M_{\mathrm{w}}=1800 \times 10^{3}$ $\mathrm{g} / \mathrm{mol}(\bullet)$. The solid blue line is $\bar{E}_{\mathrm{f}}$ (eq 3 ) for a composite film having $\bar{E}_{\mathrm{f}}=4.2 \mathrm{GPa}, \bar{E}_{\mathrm{f}}^{*}=0.1 \mathrm{GPa}$, and $\delta=2 \mathrm{~nm}$. The error bars represent one standard deviation of the data, which is taken as the experimental uncertainty of the measurement.

plateau in the apparent film modulus of $\bar{E}_{\text {f,app }} \approx 4 \mathrm{GPa}$, which corresponds to a Young's modulus of $E_{\mathrm{f}} \approx 3.6 \mathrm{GPa}$ for $v_{\mathrm{f}}=$ $0.33 .{ }^{31}$ While the values of reported moduli will be dependent on the exact processing and annealing history, we find that this value is in good agreement with results from Brillouin light scattering of PS films $\left(E_{\mathrm{f}}=3.72 \mathrm{GPa}\right)^{14}$ and bulk PS $\left(E_{\mathrm{f}}=\right.$ $3.48 \mathrm{GPa})^{32}$ but greater than results from $\operatorname{AFM}\left(E_{\mathrm{f}}=2.6 \mathrm{GPa}\right)^{33}$ and beam curvature $\left(E_{\mathrm{f}}=2.5 \mathrm{GPa}\right)^{7}$ for similar PS films. Values reported for bulk tests on PS range from 2.8 to $3.5 \mathrm{GPa} .{ }^{34}$ There is a slight disparity (but still within experimental error) in the plateau modulus for the two molecular weights studied; this is unexpected as there should be little difference in elastic modulus at such high molecular weights. We are currently exploring this issue. The second salient feature is the presence of a transition in the apparent modulus occurring for film thicknesses less than $30 \mathrm{~nm}$, with decreasing modulus for thinner films. For the thinnest films studied, the apparent modulus decreases nearly an order of magnitude compared to those of bulk PS films. ${ }^{35} \mathrm{~A}$ similar order of magnitude decrease in elastic modulus has been observed by both wafer curvature methods ${ }^{7}$ and simulations. ${ }^{8,9}$ This monotonic decrease in apparent modulus (as determined via eq 2) becomes more pronounced as the film thickness decreases. Again, this is direct evidence of a breakdown of eq 1 for the thinnest films, as was concluded from Figure 2.

To interpret these data, we propose a composite model having a surface layer of thickness $\delta$ having a modulus, $\bar{E}_{\mathrm{f}}^{*}$, different than the bulk modulus, $\bar{E}_{\mathrm{f}}$. This is a reasonable model considering that the molecular structure and dynamics at the surface of a film can be different from that in the bulk. ${ }^{2,10,11}$ The modulus of the surface layer can be either greater (hard surface) or less (soft surface) than the bulk modulus. Here, we take the thickness and modulus of the surface layer to be constant for a given material system. The effective modulus of the composite film of total thickness $h_{\mathrm{f}}$ with a surface layer of thickness $\delta$ can be obtained by the rule of mixture for bending of a plate:

$$
\begin{array}{r}
\bar{E}_{\mathrm{f}}^{\prime}=\bar{E}_{\mathrm{f}}\left(1-\frac{\delta}{h_{\mathrm{f}}}\right)^{3}+\bar{E}_{\mathrm{f}}^{*}\left(\frac{\delta}{h_{\mathrm{f}}}\right)^{3}+3 \bar{E}_{\mathrm{f}}\left(1-\frac{\delta}{h_{\mathrm{f}}}\right)\left(1-\xi-\frac{\delta}{h_{\mathrm{f}}}\right)^{2}+ \\
3 \bar{E}_{\mathrm{f}}^{*}\left(\frac{\delta}{h_{\mathrm{f}}}\right)\left(2-\xi-\frac{\delta}{h_{\mathrm{f}}}\right)^{2}
\end{array}
$$




$$
\xi=\frac{\bar{E}_{\mathrm{f}}\left(1-\frac{\delta}{h_{\mathrm{f}}}\right)^{2}+\bar{E}_{\mathrm{f}}^{*}\left(\frac{\delta}{h_{\mathrm{f}}}\right)\left(2-\frac{\delta}{h_{\mathrm{f}}}\right)}{\bar{E}_{\mathrm{f}}\left(1-\frac{\delta}{h_{\mathrm{f}}}\right)+\bar{E}_{\mathrm{f}}^{*}\left(\frac{\delta}{h_{\mathrm{f}}}\right)}
$$

This effective modulus $\left(\bar{E}_{\mathrm{f}}^{\prime}\right)$ should be used in place of $\bar{E}_{\mathrm{f}}$ in eq 1 for a composite film:

$$
\lambda_{\mathrm{e}}=2 \pi h_{\mathrm{f}}\left(\frac{\bar{E}_{\mathrm{f}}^{\prime}}{3 \bar{E}_{\mathrm{s}}}\right)^{1 / 3}
$$

Using this simple two-layer model, we use the data to fit for three parameters: the surface and bulk moduli $\left(\bar{E}_{\mathrm{f}}^{*}\right.$ and $\bar{E}_{\mathrm{f}}$, respectively) as well as the thickness of the surface layer $(\delta)$. For the data presented here, the best fit was obtained for $\bar{E}_{\mathrm{f}}^{*}=$ $0.1 \mathrm{GPa}, \bar{E}_{\mathrm{f}}=4.2 \mathrm{GPa}$, and $\delta=2 \mathrm{~nm}$. The wavelength dependence on thickness originating from this composite model can be seen in the solid blue line in Figure 2. Reasonable fits can be obtained for a surface modulus that is at least an order of magnitude less than the bulk value. The value of $\delta$ is in good agreement with the intercept of the measured wavelength vs thickness $\left(\lambda_{\mathrm{e}}=0\right)$ discussed earlier. As the films become thinner, the contribution from the surface layer becomes more pronounced; thus, the deviation from the single-layer model (eq 1) becomes larger. We recognize that this two-layer model is very simplistic and may not capture the complex polymer physics governing the material properties in ultrathin polymer films. For example, Torkelson and co-workers ${ }^{36}$ have shown that there is a distribution of $T_{\mathrm{g}} \mathrm{s}$ propagating away from the free surface in nanoconfined films and have proposed a gradient model to describe the dynamics of a fluorescent probe in ultrathin films. The simple two-layer composite model (eq 5) appears to be a reasonable first approximation that accounts for the deviations in the observed buckling wavelength as a function of film thickness, but this does not preclude more complex models from being entertained in the future.

Similar to the wavelength data, the composite model (eq 3) can be invoked to predict the decrease in modulus in ultrathin films. The solid blue line in Figure 3 corresponds to the composite bending modulus $\left(\bar{E}_{\mathrm{f}}^{\prime}\right)$ predicted by eq 3 for a twolayer model with the same properties deduced earlier. The composite model appropriately predicts the decrease in apparent modulus observed for $h_{\mathrm{f}}<40 \mathrm{~nm}$. Several previous studies have suggested the presence of a mobile, rubberlike surface on PS films ${ }^{2,37-39}$ with a thickness estimated to be less than $5 \mathrm{~nm}$, which is in qualitative agreement with the results from our composite model. Additionally, molecular dynamics simulations show a surface region of enhanced mobility that is $1.5 \mathrm{~nm}$ thick, ${ }^{11}$ in good agreement with our results using the composite model. Recall from eq 1 that the wavelength is proportional to the polymer film thickness. Thus, for thick films, the wavelength (and thus the apparent modulus) is not sensitive to the small surface region. However, for the thinnest film, this surface region corresponds to near $40 \%$ of the total film thickness. It is in this region that we are most sensitive to the surface. For films thicker than $30 \mathrm{~nm}$, this surface layer still exists, but the contribution is negligible.

To test the broader applicability of this composite model, we also examined the mechanical response of PMMA ultrathin films. Unlike PS films, the segmental mobility for supported PMMA ultrathin films has been found to decrease due to the attractive interaction between the methacrylate moiety and the oxide layer on a silicon substrate. ${ }^{40-42}$ However, for a non-

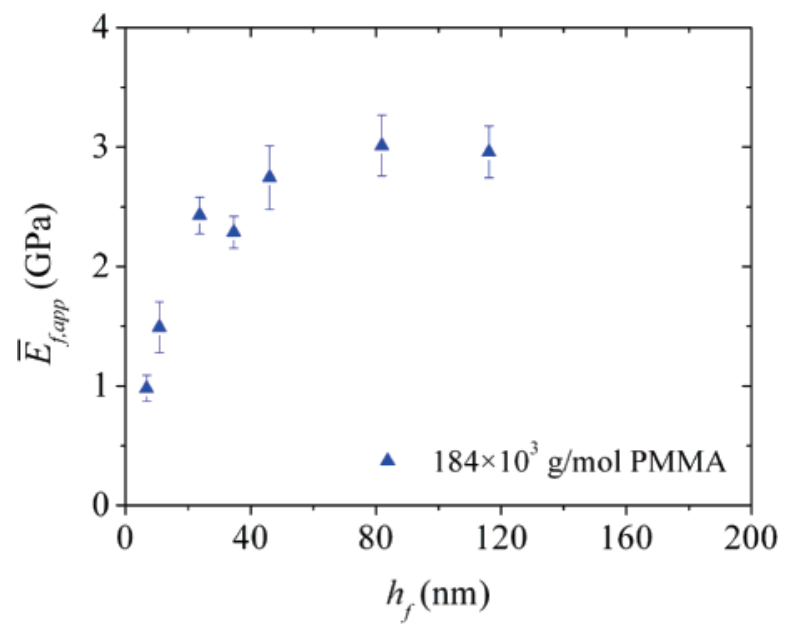

Figure 4. Apparent modulus $\left(\bar{E}_{\mathrm{f}, \mathrm{app}}\right)$ as a function of thickness $\left(h_{\mathrm{f}}\right)$ for PMMA films having $M_{\mathrm{w}}=184 \times 10^{3} \mathrm{~g} / \mathrm{mol}$. The PMMA films display a decrease in the apparent modulus similar to the PS films. The error bars represent one standard deviation of the data, which is taken as the experimental uncertainty of the measurement.

attractive substrate, there is a net increase in segmental mobility, which has been similarly attributed to a region of enhanced mobility at the free surface, although a much weaker thickness dependence is typically observed compared to PS thin films. ${ }^{40,41,43,44}$ For PMMA on PDMS, the substrate should be relatively noninteracting, and thus the buckling should be sensitive to the mobile surface layer, allowing us to assess the functional form and consistency of our two-layer model. Figure 4 illustrates the film thickness dependence of the moduli of thin PMMA films. A similar trend is observed to the PS thin films described previously as there is a decrease in the apparent modulus for films less than $40 \mathrm{~nm}$ thick. The modulus decrease is nearly $30 \%$ from the bulk, which is greater than expected on the basis of the minor $T_{\mathrm{g}}$ dependence of ultrathin PMMA films. ${ }^{40,44}$ However, the depression in the modulus for both PS and PMMA is consistent with the predictions of de Pablo and co-workers, ${ }^{8,9}$ which indicate a reduced modulus $\left(\bar{E}_{\mathrm{f}, \text { app }} / \bar{E}_{\text {bulk }}\right)$ that decreases as the feature size decreases with a transition occurring at $\approx 40 \mathrm{~nm}$ for amorphous polymer films. It is interesting to note that the simulations also predict a free surface layer whose properties deviate from that of the bulk material. Our proposed model to fit the data is consistent with these simulations. To compare our data to these predictions, the data from Figures 2 and 4 are replotted in terms of this reduced modulus. The bulk modulus is taken as the average of the plateau region in the film thickness data. As shown in Figure 5 , the reduced modulus is polymer-independent and decreases for thin films; both results are consistent with the simulations. Future work will focus on the universality of this behavior as well as the temperature dependence of the transition from bulk behavior as a function of proximity to bulk $T_{\mathrm{g}}$.

Conclusions. We offer a new method for probing the elastic moduli of ultrathin amorphous polymer films. In our bucklingbased metrology, the modulus-dependent wavelength of the buckling instability can be interpreted using established buckling mechanics. However, the apparent modulus deviates from bulk for films less than $40 \mathrm{~nm}$ thick for both PS and PMMA systems. The mechanical property data are consistent with previous literature results on supported ultrathin films having a free surface as measured by BLS, wafer curvature experiments, and molecular dynamics simulations. An analytical solution for a composite model comprised of a soft surface layer of finite thickness and the remainder of the film displaying bulk modulus 


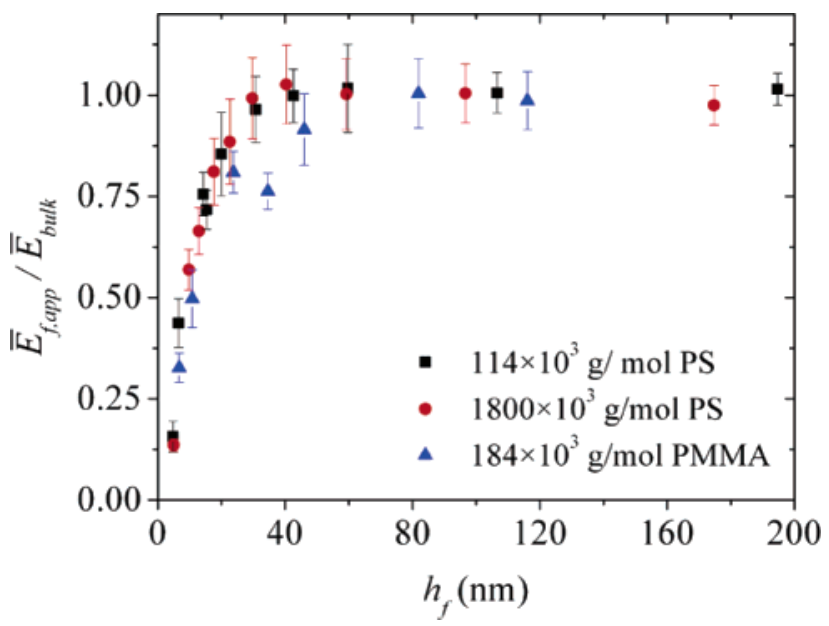

Figure 5. Reduced modulus $\left(\bar{E}_{\text {app }} / \bar{E}_{\text {bulk }}\right)$ for the two PS $(\mathbf{\square}, \mathbf{0})$ and PMMA $(\mathbf{\Lambda})$ materials as a function of thickness $\left(h_{\mathrm{f}}\right)$. The error bars represent one standard deviation of the data, which is taken as the experimental uncertainty of the measurement.

accurately describes the data. The decrease in the apparent modulus is compelling and highlights fabrication and stability issues for sub-40 $\mathrm{nm}$ features patterned in thin polymer films.

Acknowledgment. We thank T. P. Russell, G. B. McKenna, M. J. Fasolka, W. L. Wu, and C. L. Soles for fruitful discussions. C.M.S., B.D.V., and C.H. gratefully acknowledge the NIST National Research Council Postdoctoral Fellowship Program. R.H. acknowledges support from the National Science Foundation through Grant CMS-0547409. This manuscript is an official contribution of the National Institute of Standards and Technology.

Supporting Information Available: Plot of $\bar{E}_{\mathrm{f} \text {,app }}$ as a function of $h_{\mathrm{f}}$ for PS films for different Poisson's ratios, $v_{\mathrm{f}}$. This material is available free of charge via the Internet at http://pubs.acs.org.

\section{References and Notes}

(1) Alcoutlabi, M.; McKenna, G. B. J. Phys.: Condens. Matter 2005, 17, R461-R524.

(2) Forrest, J. A.; Dalnoki-Veress, K. Adv. Colloid Interface Sci. 2001 94, 167-196.

(3) Stoykovich, M. P.; Cao, H. B.; Yoshimoto, K.; Ocala, L. E.; Nealey, P. F. Adv. Mater. 2003, 15, 1180-1184.

(4) VanLandingham, M. R.; Villarrubia, J. S.; Guthrie, W. F.; Meyers, G. F. Macromol. Symp. 2001, 167, 15-43.

(5) Strojny, A.; Xia, X. Y.; Tsou, A.; Gerberich, W. W. J. Adhes. Sci. Technol. 1998, 12, 1299-1321.

(6) Every, A. G. Meas. Sci. Technol. 2002, 13, R21-R39.

(7) Zhao, J. H.; Kiene, M.; Hu, C.; Ho, P. S. Appl. Phys. Lett. 2000, 77, $2843-2845$

(8) Böhme, T. R.; de Pablo, J. J. J. Chem. Phys. 2002, 116, 9939-9951.

(9) Van Workum, K.; de Pablo, J. J. Nano Lett. 2003, 3, 1405-1410.

(10) Yoshimoto, K.; Jain, T. S.; Nealey, P. F.; de Pablo, J. J. J. Chem. Phys. 2005, 122, 144712.

(11) Mansfield, K. F.; Theodorou, D. N. Macromolecules 1991, 24, 62836294.

(12) O'Connell, P. A.; McKenna, G. B. Science 2005, 307, 1760.

(13) Shulha, H.; Kovalev, A.; Myshkin, N.; Tsukruk, V. V. Eur. Polym. J. 2004, 40, 949-956.

(14) Sun, L.; Dutcher, J. R.; Giovannini, L.; Nizzoli, F.; Stevens, J. R.; Ord, J. L. J. Appl. Phys. 1994, 75, 7482-7488.
(15) Hartschuh, R.; Ding, Y.; Roh, J. H.; Kisliuk, A.; Sokolov, A. P.; Soles, C. L.; Jones, R. J.; Hu, T. J.; Wu, W. L.; Mahorowala, A. P. J. Polym. Sci., Part B: Polym. Phys. 2004, 42, 1106-1113.

(16) Forrest, J. A.; Dalnoki-Veress, K.; Dutcher, J. R. Phys. Rev. E 1998, $58,6109-6114$

(17) Forrest, J. A.; Rowat, A. C.; Dalnoki-Veress, K.; Stevens, J. R.; Dutcher, J. R. J. Polym. Sci., Part B: Polym. Phys. 1996, 34, 30093016.

(18) Stafford, C. M.; Harrison, C.; Beers, K. L.; Karim, A.; Amis, E. J.; VanLandingham, M. R.; Kim, H. C.; Volksen, W.; Miller, R. D.; Simonyi, E. E. Nat. Mater. 2004, 3, 545-550.

(19) Allen, H. G. Analysis and Design of Structural Sandwich Panels; Pergamon: New York, 1969

(20) Groenewald, J. Physica A 2001, 298, 32-45.

(21) Huang, R. J. Mech. Phys. Solids 2005, 53, 63-89.

(22) Huang, Z. Y.; Hong, W.; Suo, Z. J. Mech. Phys. Solids 2005, 53, 2101-2118

(23) Mark, J. E. Physical Properties of Polymers Handbook; American Institute of Physics: New York, 1996.

(24) Certain equipment, instruments or materials are identified in this paper in order to adequately specify the experimental details. Such identification does not imply recommendation by the National Institute of Standards and Technology nor does it imply the materials are necessarily the best available for the purpose.

(25) Wallace, W. E.; van Zanten, J. H.; Wu, W. L. Phys. Rev. E 1995, 52, R3329-R3332.

(26) Russell, T. P. Mater. Sci. Rep. 1990, 5, 171-271.

(27) We noticed a slight dependence of the film roughness on spin speed. At $1000 \mathrm{rpm}$, the roughness was approximately $1.0-1.5 \mathrm{~nm}$, and at $2000 \mathrm{rpm}$ the roughness was approximately $0.7-1.0 \mathrm{~nm}$, while at 4000 $\mathrm{rpm}$ the roughness was approximately $0.5-0.7 \mathrm{~nm}$.

(28) Stafford, C. M.; Guo, S.; Harrison, C.; Chiang, M. Y. M. Rev. Sci. Instrum. 2005, 76, 062207.

(29) Pedersen, J. S.; Schurtenberger, P. Europhys. Lett. 1999, 45, 666672.

(30) Aharoni, S. M. Macromolecules 1983, 16, 1722-1728.

(31) Brandrup, J.; Immergut, E. H.; Grulke, E. A. Polymer Handbook; Wiley-Interscience: New York, 1999.

(32) Lindsay, S. M.; Halawith, B.; Patterson, G. D. J. Polym. Sci., Part C: Polym. Lett. 1982, 20, 583-588.

(33) Du, B.; Tsui, O. K. C.; Zhang, Q.; He, T. Langmuir 2001, 17, 32863291.

(34) Fried, J. R. Polymer Science and Technology; Prentice Hall: Englewood Cliffs, NJ, 1995.

(35) We assume a constant Poisson's ratio $\left(v_{\mathrm{f}}=0.33\right)$ for all film thicknesses, which is a current topic of debate. However, if we calculate the apparent modulus using the realistic extremes in Poisson's ratio $\left(0<v_{\mathrm{f}}<0.5\right)$, the magnitude of the decrease in apparent modulus in ultrathin polymer films is still exhibited (see figure in Supporting Information).

(36) Ellison, C. J.; Torkelson, J. M. Nat. Mater. 2003, 2, 695-700.

(37) Fischer, H. Macromolecules 2002, 35, 3592-3595.

(38) Sasaki, T.; Shimizu, A.; Mourey, T. H.; Thurau, C. T.; Ediger, M. D. J. Chem. Phys. 2003, 119, 8730-8735.

(39) Keddie, J. L.; Jones, R. A. L.; Cory, R. A. Europhys. Lett. 1994, 27 $59-64$

(40) Keddie, J. L.; Jones, R. A. L.; Cory, R. A. Faraday Discuss. 1994, 219-230.

(41) Fryer, D. S.; Peters, R. D.; Kim, E. J.; Tomaszewski, J. E.; de Pablo, J. J.; Nealey, P. F.; White, C. C.; Wu, W. L. Macromolecules 2001, 34, 5627-5634.

(42) Priestley, R. D.; Broadbelt, L. J.; Torkelson, J. M. Macromolecules 2005, 38, 654-657.

(43) Roth, C. B.; Dutcher, J. R. Eur. Phys. J. E 2003, 12, S103-S107.

(44) Sharp, J. S.; Forrest, J. A. Phys. Rev. E 2003, 67, 031805.

(45) The uncertainty in the film thickness measurement by XR is approximately $\pm 0.1 \mathrm{~nm}$ for $h_{\mathrm{f}}<10 \mathrm{~nm}$ and increases to approximately $\pm 0.5 \mathrm{~nm}$ for the thickest films examined. This uncertainty arises primarily from the goodness of the data fit related to the $q$ resolution and number of data points per Kessig fringe. This uncertainty in thickness was accounted for when calculating the overall uncertainty in the measurement via error propagation.

MA060790I 